\title{
A peculiar uncommon cutaneous neoplasia: Angiosarcoma of the head and neck of the elderly (Wilson Jones Angiosarcoma)
}

\author{
Alessandro D'Amuri*1 ${ }^{* 1}$ Federica Floccari ${ }^{2}$, Raffaele Filotico ${ }^{3}$, Marcello Filotico ${ }^{4}$ \\ ${ }^{1}$ Anatomic Pathology Unit, "A. Perrino" Hospital, Brindisi, Italy \\ ${ }^{2}$ Clinic Pathology Unit, "L. Bonomo" Hospital, Andria (BAT), Italy \\ ${ }^{3}$ Dermatology and Melanoma Unit, "A. Perrino" Hospital, Brindisi, Italy \\ ${ }^{4}$ Anatomic Pathology Unit, "Card. G. Panico" Hospital, Tricase (LE), Italy
}

Received: February 10, 2019

Accepted: May 26, 2019

Online Published: July 19, 2019

DOI: $10.5430 /$ crcp.v6n1p9

URL: https://doi.org/10.5430/crcp.v6n1p9

\begin{abstract}
Cutaneous Angiosarcoma of the elderly's face is a rare and peculiar neoplasm with all its own clinical and morphological characteristics. The observation of a classic case in the clinical presentation and morphology has offered the opportunity to revisit this topic.
\end{abstract}

Key Words: Wilson Jones Angiosarcoma, Cutaneous angiosarcoma, Imunohistochemistry

\section{INTRODUCTION}

A 85 years old man has few months erythematous patch on the right cheek which has progressively expanded, reaching the scalp and the preauricular region. Lesion continued to expand despite various local therapies. Histological examination shows the dermal level numerous cavities with an irregular lumen, sometimes wide, other times fissuriform in which papillary vegetations often protrude. Lumen of the cavities, as well as the axes of the papillary vegetations are papered by plump endothelial elements, hyperchromatic. These elements express intensely and extensively CD34 and CD31. Further studies also showed positivity for podoplanin, p53, c-myc, and negativity for HHV8. On the basis of these elements, the diagnosis of "Well-Differentiated $\mathrm{Cu}$ taneous Angiosarcoma of the head and neck of the elderly" is posed. Two aspects characterize this neoplasia: the localization to the cheek with extension to the scalp, to the retroauricular region and the neck, the advanced age of the patient who is usually male. Wide variety of histological aspects can be found both in the various neoplasms and in those of the same tumor. From highly differentiated lesions hemangioma/lymphangioma-like to classically papillary patterns, to solid poorly differentiated, epithelioid or spindle cell. In these solid lesions the differential diagnosis from carcinoma, melanoma, or atypical fibroxanthoma can be difficult.

\section{CASE REPORT}

A 85 years old man has a few months erythematous patch on the right cheek, which has progressively expanded, reaching the scalp and the preauricular region. Upon palpation, the lesion is infiltrated and hardened. The tumor continued to expand despite various local therapies (see Figures $1 \mathrm{a}, \mathrm{b}$ ).

*Correspondence: Alessandro D’Amuri; Email: a.damuri@inwind.it; Address: Anatomic Pathology Unit, "A. Perrino" Hospital, Brindisi, Italy. 


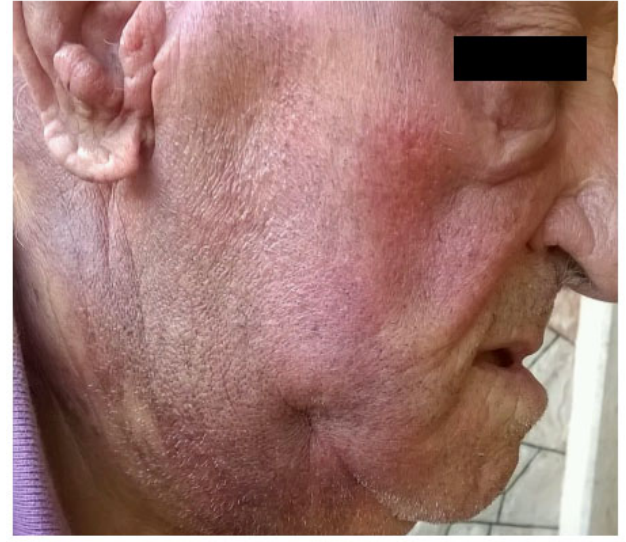

(a)

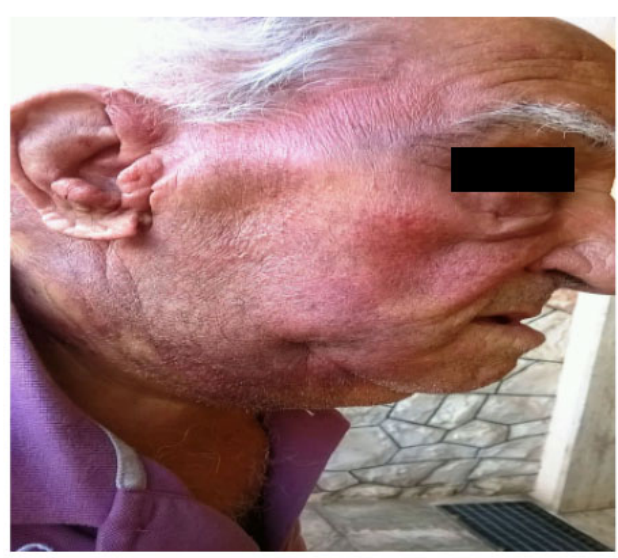

(b)

Figure 1. (a) and (b). Erythematous area interesting the cheek, preauricolar, laterocervical region and the initial part of the scalp

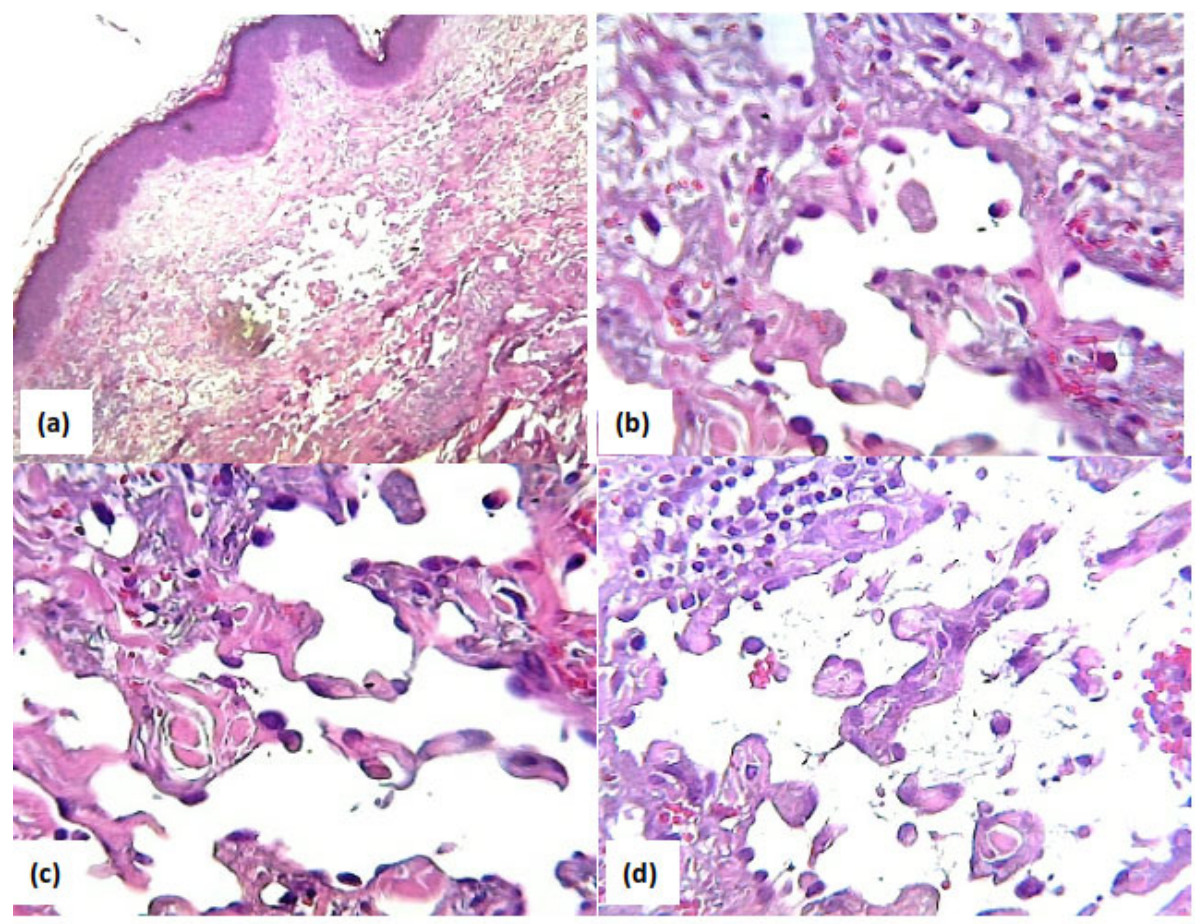

Figure 2. (a) Large irregularly shaped vascular spaces occupy a large part of the dermis (HE 40x); (b), (c), (d) vascular cavities with intraluminal papillary vegetations (HE 120x).

An incisional biopsy of a cutaneous lozenge is performed. fissuriform in which papillary vegetations often protrude (see The bioptic fragment is fixed in formalin and in paraffin em- Figures 2 (a), (b), (c) and (d)). The lumen of the cavities, bedded. Sections stained with Hematoxilyn-Eosin (HE) and subjected to immunohistochemical investigation with CD34 and CD31 antibodies. Histological observation reveals the complete integrity of the epidermal lining. The dermis, on the other hand, appears to be dissociated by numerous cavities with an irregular lumen, sometimes wide, other times as well as the axes of the papillary vegetations, is coated by plump endothelial elements, hyperchromatic, distinctly atypical (see Figures 3 (a), (b), (c) and (d)). These elements express intensely and extensively CD34 (see Figures 4(a) and (b)) and CD31(see Figures 4 (c) and (d)). On the basis of these elements the diagnosis of "Well-Differentiated 
Cutaneous Angiosarcoma of the head and neck of the el- and c-myc; and subsequently treated only with gemcitabine derly" is made. The patient for further investigation and $\left(1,000 \mathrm{mg} / \mathrm{m}^{2}\right.$ i.v. every week for 3 weeks every 4 weeks). treatment is admitted to the National Cancer Institute (INT) No local radiotherapy (RT) treatment was performed. CXR, in Milan (Italy), where the histological diagnosis is con- CT of the head, neck and chest have been negative. The firmed, and further validated with other immunohistochemi- patient was free of disease at the 15-month follow-up. cal and biomolecular researches for podoplanin, HHV8, p53

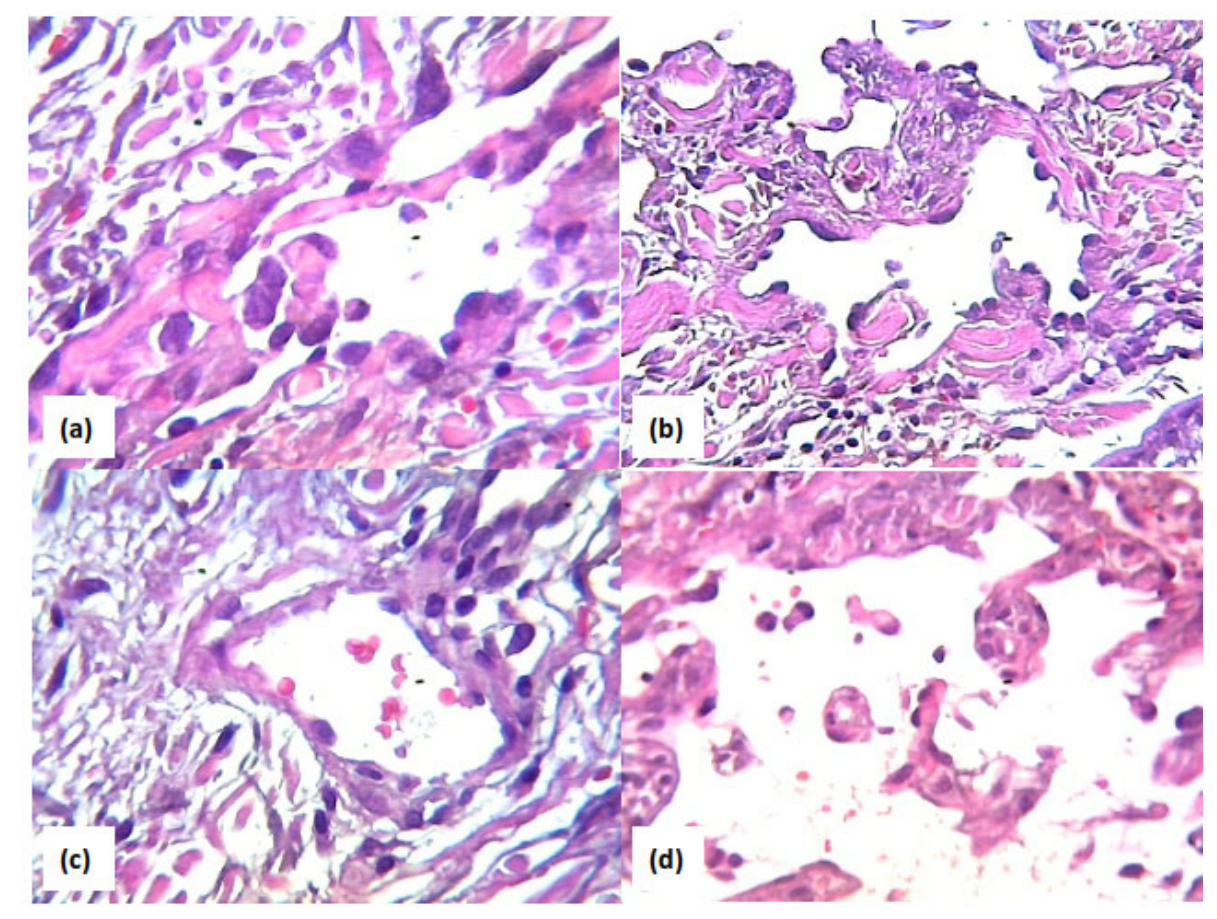

Figure 3. (a), (b), (c), (d) Plump and atypical endothelial cells delimit the lumen of the vascular cavities (HE 120x).

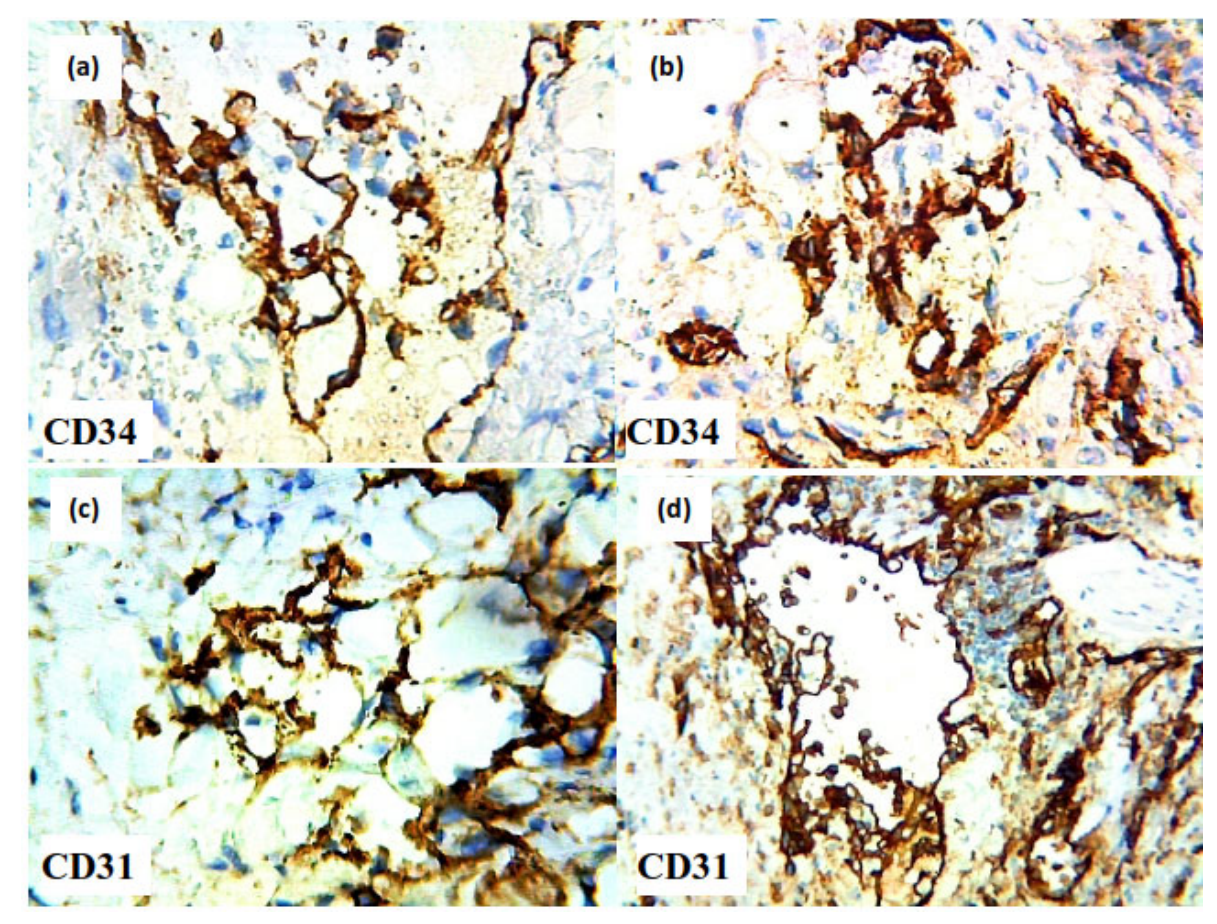

Figure 4. (a), (b) CD 34 (40x); (c), (d) CD31 (40x). 


\section{Discussion AND Conclusions}

In 1964 Wilson Jones described in detail this particular cutaneous angiosarcoma variant which is characterized by its particular clinical presentation. ${ }^{[1]}$ Two aspects characterize this neoplasia: the localization to the cheek with extension to the scalp, to the retroauricular region and the neck, the advanced age of the patient who is usually male. ${ }^{[2]}$ The tumor begins as an erythematous macula which progressively extends in a centrifugal manner giving the skin an edematous consistency. As the lesion progresses, the color pass through violet to bluish assuming sometimes the appearance of a hematoma. The plaque is progressively hardened and nodules may appear in its context and undergo ulceration. The centrifugal progress of the neoplasia is often very rapid so that in a short time the whole cheek is occupied.$^{[3]}$ Histopathological investigation reveals the neoformation is more extensive than would appear on clinical examination. ${ }^{[4]}$ From highly differentiated lesions hemangioma/lymphangioma-like to classically papillary patterns, to solid poorly differentiated epithelioid or spindle cell. In these solid lesions the differential diagnosis from carcinoma, melanoma, or atypical fibroxanthoma can be difficult. ${ }^{[5,6,9]}$ For these tumors, the literature reports the following immunohistochemical panel (Erg +, claudin $5+$, vimentin +, CD31 +, FL1 +, D2-40 +, VEGFR variable, Keratin variable, CD34 +/-, prox 1+/-, vWF $-/+$, EMA - and S-100 -). ${ }^{[7,11,12]}$ In this particular neoplasia to date, no characteristic chromosomal aberration has been signaled. According to Manner et al. in post-irradiation skin angiosarcomas or associated with lymphedema, an amplification of the MYC gene would occur, which would not occur in primitive angiosarcomas. ${ }^{[8,10]}$ Requena et al. confirmed that experienced the MYC amplifications in angiosarcoma, as detected by either FISH or immunohistochemistry, they are much more widespread in angiosarcomas than originally suggested by Manner J. Remarkably, so far, almost all of our facial and scalp angiosarcoma cases have stained positive for MYC. We, therefore, suggest including immunohistochemical MYC staining in any diagnostic vascular antibody panel. Immunohistochemical MYC staining is qualitatively equivalent to analogous FISH. ${ }^{[3]}$

\section{CONFLiCTS OF INTEREST Disclosure}

The authors declare that they have no competing interest.

\section{REFERENCES}

[1] Wilson Jones E. Malignant angioendothelioma of the skin. Br J Dermatol. 1964; 76: 21-39. PMid:14112957. https://doi.org/10.1 111/j.1365-2133.1964.tb13970.x

[2] Hodgkinson DJ, Soule EH, Woods JE. Cutaneous angiosarcoma of the head and neck. Cancer. 1979; 44: 1106-1113. https://doi .org/10.1002/1097-0142(197909) 44: 3<1106: :AID-CNCR2820440345>3.0.C0;2-C

[3] Requena L, Kutzner H. Cutaneous Soft Tissue Tumors (position in Kindle 21087-21088), Wolters Kluwer Philadelphia, PA 1 USA, Kindle Edition, 2015.

[4] Rosai J, Sumner HW, Major MC, et al. Angiosarcoma of the skin. A clinicopathologic and fine structural study. Hum Pathol. 1976; 7: 83109. https://doi .org/10.1016/S0046-8177 (76)80007-X

[5] Wilson Jones E. Malignant vascular tumors. Clin Exp Dermatol. 1976; 1: 287-312. https://doi.org/10.1111/j.1365-2230. 1976.tb01435. $\mathrm{x}$

[6] Enzinger FM, Weiss SW. Malignant vascular tumors. In: Soft Tissue Tumors. 3rd ed. St. Louis, MO: Mosby. 1997: 641-677.

[7] Lin F, Prichard J. Handbook of Practical Immunohistochemistry: Frequently Asked Questions (position in Kindle 31712-31715), 2nd ed. Springer New York. Kindle edition 2015.
[8] Manner J, Radlwimmer B, Hohenberger P, et al. MYC high level gene amplification is a distinctive feature of angiosarcomas after irradiation or chronic lymphedema. Am J Pathol. 2010; 176: 34-39. PMid:20008140. https://doi.org/10.2353/ajpath.2010.09 0637

[9] Das Gupta M, Chakrabarti N, Agrawal P, et al. Angiosarcoma of the scalp. Indian J Plast Surg. 2009; 42(1): 118-121. PMid:19881033. https://doi .org/10.4103/0970-0358.53023

[10] Glickstein J, Sebelik ME, Lu Q. Cutaneous Angiosarcoma of the Head and Neck: A Case Presentation and Review of the Literature. Ear, Nose \& Throat Journal. 2006; 85(10): 672-4. https : //doi .org/10.1177/014556130608501018

[11] Wang L, Lao IW, Yu L, et al. Clinicopathological features and prognostic factors in angiosarcoma: A retrospective analysis of $200 \mathrm{pa}-$ tients from a single Chinese medical institute. Oncol Lett. 2017; 14(5): 5370-78.

[12] Cabral ANF, Rocha RH, Amaral ACVD, et al. Cutaneous angiosarcoma: report of three different and typical cases admitted in a unique dermatology clinic. An Bras Dermatol. 2017; 92(2): 235238. PMid:28538886. https ://doi .org/10.1590/abd1806-4 841.20175326 